\title{
Medullary carcinoma of the thyroid metastatic to breast
}

\author{
N Kiely, N Williams, G Wilson, RJ Williams
}

\begin{abstract}
Summary
Medullary carcinoma of the thyroid commonly spreads to the lymphatics and later on in the disease process, to distant sites such as lung, liver and bone. Spread to the breast is rare (only two previous reported cases) and presents a major diagnostic dilemma. We report a case of metastatic medullary carcinoma of the thyroid which presented as a primary breast nodule with ipsilateral axillary lymphadenopathy. Clearly it was important to distinguish this tumour from a breast primary as each are managed differently. Both cytological and radiological investigations were inconclusive but excision biopsy was diagnostic.
\end{abstract}

Keywords: medullary carcinoma, thyroid, metastasis, breast

Medullary carcinoma of the thyroid (MCT) commonly metastasises early to local lymph nodes and later distantly to the lungs and liver. ${ }^{1,2}$ Other sites of distant metastases are rare. We report a case of an MCT metastasis to the breast which presented as a primary breast nodule. This case illustrates a rare site for thyroid metastasis and the diagnostic difficulties encountered.

\section{Case report}

A 64-year-old woman presented in 1992 with an asymptomatic left-sided neck swelling. Examination revealed an enlarged thyroid with a palpable right upper cervical lymph node and she therefore underwent total thyroidectomy and bilateral cervical node excision. Histology and immunohistochemistry of the resected specimen confirmed a medullary carcinoma.

She re-presented in 1993 whilst under active follow-up with a nodule in the right posterior triangle. Excision biopsy confirmed recurrent tumour (MCT) and radiotherapy was therefore given. In 1994, a further nodule appeared in the left posterior triangle. However, at this time she also discovered a lump in the left breast and a 2-cm diameter soft, tender mass in the left axillary tail and axillary lymphadenopathy was noted. Fine needle aspiration for cytology of the breast lump was able to confirm the presence of malignant epithelial cells, but could not determine the tissue of origin in the absence of tissue architecture. ${ }^{131}$ I-Meta-iodobenzyl- guanidine (MIBG) scan demonstrated increased uptake in the cervical lymph nodes but none in the breast or axillae. Ultrasonography suggested the breast lesion to be a primary breast carcinoma. Computed tomography (CT) scan confirmed the presence of axillary lymph nodes and the breast lesion, but excluded intrathoracic or abdominal disease. She therefore underwent wide local exision of the breast lump with axillary clearance. Paraffin sections and immunohistochemistry revealed the breast lump to be a metastatic medullary carcinoma of the thyroid with no axillary lymph node involvement. She now awaits a functional neck dissection.

\section{Discussion}

Metastases in MCT are common and occur both by haematogenous and lymphatic spread. ${ }^{1}$ Regional lymph node involvement occurs early (affecting approximately $60 \%$ of patients ${ }^{1}$ ) and later on in the disease process distant metastases are found in up to $40 \%$ of patients. ${ }^{1,2}$ The commonest sites of distant spread are lung, liver and bone. ${ }^{1}$ Rarer sites include brain and skin. ${ }^{1,3,4}$

Breast metastases from MCT are very rare with only two other cases reported in the literature. Ordonez et al describe a 72-year-old woman with a 22-year history of MCT who presented with regional and cutaneous metastases and later developed a $5-\mathrm{cm}$ breast mass and concurrent axillary lymphadenopathy. ${ }^{4}$ Fine needle aspiration biopsy revealed a metastatic MCT. Following a simple mastectomy the patient died some months later. Ahuja and Ernst $^{3}$ report a 47-year-old woman with a 15-year history of MCT. She presented with diarrhoea and metastases to bone and lung. Six years later, she presented with multiple bilateral intramammary lesions, diagnosed as metastatic MCT by excision biopsy. The patient died of carcinomatosis nine years later.

\section{Learning points}

medullary carcinoma rarely metastasises to breast

- 'primary' breast lesions may rarely be metastatic

- fine needle aspiration for cytological assessment cannot always determine the tissue of origin 
Our case report differs in that the patient was asymptomatic on presentation and developed the breast lesion relatively soon after the diagnosis. In addition no other distant lesion has yet been detected. An accurate diagnosis of the breast mass was difficult to establish as fine needle aspiration biopsy was inconclusive, and radiological investigations suggested a primary breast lesion. ${ }^{131}$ I-MIBG scanning can be ineffective in localising metastatic MCT. ${ }^{5}$ In one study of 10 patients with biochemical evidence

1 Høire J, Jørgensen O, Stenwig A, Langmark F. Medullary thyroid carcinoma in Norway. Acta Chir Scand 1988; 154: 339-43.

2 Buhr H, Kallinowski F, Raue F, Frank-Raue K, Herfarth C. Buhr H, Kallinowski F, Raue F, Frank-Raue K, Herfarth C.
Microsurgical neck dissection for occult metastasising Microsurgical neck dissection for occult metastasising 3 medullary thyroid carcinoma. Cancer 1993; 72: 3685-93. 3 Ahuja S, Ernst H. Dormant metastases in medullary thyroid carcinoma of recurrence after surgery only one lesion was accurately identified by ${ }^{131}$ I-MIBG scan. ${ }^{5}$ This not only has implications for diagnosis but also for ${ }^{131}$ I treatment of metastases.

In conclusion, therefore, although MCT commonly metastasises to local and distant sites, spread to the breast is very rare, and should be considered a diagnostic possibility in patients with a past history of MCT presenting with an apparently primary breast lesion.

4 Ordonez N, Katz R, Luna M, Samaan N. Medullary thryoid carcinoma metastatic to breast diagnosed by fine needle aspiration biopsy. Diagn Cytopathol 1988; 4: 254-7.

5 Cabezas R, Berna L, Estorch M, Carrio I, Garcia-Ameijeiras A. Localisation of metastases from medullary thyroid carcinoma using different methods. Henry Ford Hosp $f$ 1989; 37: 169-72.

\title{
Bacterial meningitis after $M M R$ immunisation
}

\author{
FAI Riordan, JA Sills, APJ Thomson, CA Hart
}

\begin{abstract}
Summary
Two children developed bacterial meningitis within five days of measles-mumpsrubella (MMR) immunisation. Diagnosis was delayed because symptoms were attributed to the vaccine, although both had a raised C-reactive protein. Fever or rash within five days of $M M R$ vaccination are unlikely to be due to the vaccine and a raised C-reactive protein suggests bacterial infection.
\end{abstract}

Keywords: bacterial meningitis, measles-mumpsrubella vaccination, $\mathrm{C}$-reactive protein

Children may develop fever and rash 7-12 days after immunisation with measles-mumpsrubella (MMR) vaccine. ${ }^{1}$ The development of fever and rash before this may be due to an underlying bacterial infection. We report two children who developed bacterial meningitis shortly after MMR immunisation, but in whom the early symptoms were ascribed to the vaccine.

\section{Case 1}

Two days after MMR immunisation a 13month-old girl developed fever and a generalised 'blotchy red rash'. She was seen by a general practitioner who prescribed an antihistamine for a possible vaccine reaction. The rash faded, but the child was increasingly lethargic. She was taken to the local hospital and admitted. On admission she had a fever of $38.8^{\circ} \mathrm{C}$ and a red throat, but no other localising signs of infection. The peripheral white cell count was
$9.8 \times 10^{9} / 1$ (granulocytes $75 \%$ ) and C-reactive protein was $235.6 \mathrm{mg} / 1$ (normal $<8 \mathrm{mg} / \mathrm{l}$ ).

Six hours after admission neck stiffness and a sparse petechial rash were noted. Lumbar puncture produced turbid cerebrospinal fluid (CSF) which grew Neisseria meningitidis. The child was treated with antibiotics and made a full recovery.

\section{Case 2}

A one-year-old girl became lethargic and febrile three days after MMR immunisation. She was seen by her general practitioner who diagnosed a 'viral illness', amd prescribed paracetamol. The following morning she remained lethargic and developed a 'blotchy red rash' on her legs. She was taken to an Accident and Emergency department where her symptoms were thought to be due to her recent immunisation. She was discharged, but returned six hours later because of increasing lethargy, fever and pallor. By this time she had marked neck stiffness, a bulging anterior fontanelle, and was poorly perfused with a sparse petechial rash on her legs. A full blood count showed a white cell count of $12.5 \times 10^{9} / 1(78 \%$ neutrophils) and serum C-reactive protein was $193 \mathrm{mg} / \mathrm{l}$. She required initial resuscitation with plasma expansion and was tranferred to the intensive care unit for stabilisation.

Lumbar puncture was deferred until the next day when CSF showed a white blood cell count of $4 \times 10^{9} / 1$ ( $85 \%$ neutrophils), protein $0.85 \mathrm{~g} / 1$ and CSF/blood glucose ratio 0.4. Bacterial and viral cultures and antigen studies on blood and CSF were all negative. She received antibiotics for seven days and made a full recovery. 